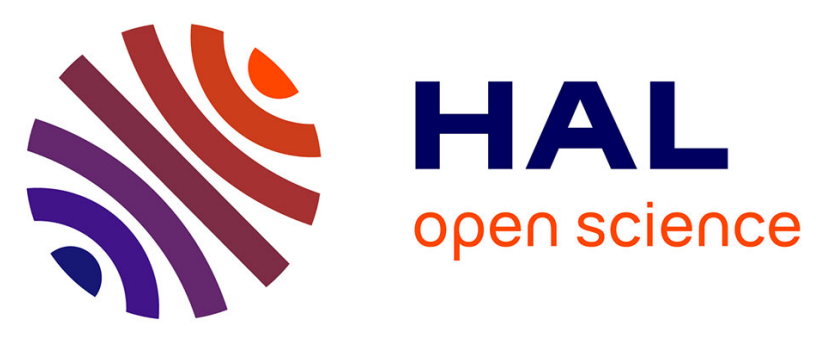

\title{
Differentiating between active and latent tuberculosis with chest computed tomography
}

Sébastien Bommart, Jérémy Charriot, Nicolas Nagot, Hélène

Vernhet-Kovacsik, Marie Revel, Clément Boissin, Arnaud Bourdin, Edouard

Tuaillon

\section{To cite this version:}

Sébastien Bommart, Jérémy Charriot, Nicolas Nagot, Hélène Vernhet-Kovacsik, Marie Revel, et al.. Differentiating between active and latent tuberculosis with chest computed tomography. Diagnostic and Interventional Imaging, In press, 10.1016/j.diii.2021.05.011 . hal-03274653

\section{HAL Id: hal-03274653 \\ https://hal.science/hal-03274653}

Submitted on 30 Jun 2021

HAL is a multi-disciplinary open access archive for the deposit and dissemination of scientific research documents, whether they are published or not. The documents may come from teaching and research institutions in France or abroad, or from public or private research centers.
L'archive ouverte pluridisciplinaire HAL, est destinée au dépôt et à la diffusion de documents scientifiques de niveau recherche, publiés ou non, émanant des établissements d'enseignement et de recherche français ou étrangers, des laboratoires publics ou privés. 


\title{
Differentiating between active and latent tuberculosis with chest computed tomography
}

\author{
Sébastien Bommart ${ }^{\mathrm{a}, \mathrm{b}, *}$, Jeremy Charriot $^{\mathrm{b}, \mathrm{c}}$, Nicolas Nagot ${ }^{\mathrm{d}}$, Hélène Vernhet-Kovacsik ${ }^{\mathrm{a}}$, \\ Marie P. Revel ${ }^{\mathrm{e}, \mathrm{f}}$, Clément Boissin ${ }^{\mathrm{c}}$, Arnaud Bourdin ${ }^{\mathrm{b}, \mathrm{c}}$, Edouard Tuaillon ${ }^{\mathrm{g}, \mathrm{h}}$ \\ a Department of Radiology, CHU Montpellier, avenue Doyen Gaston Giraud, 34000 Montpellier, France \\ b PhyMedExp Inserm U1046, UMR9214 CNRS, 34000 Montpellier, France \\ ${ }^{\mathrm{c}}$ Department of Respiratory Diseases, CHU Montpellier, 34000 Montpellier, France \\ d Department of Biostatistics, CHU Montpellier, 34000 Montpellier, France \\ e Department of Radiology, Cochin Hospital, Assistance Publique-Hopitaux de Paris, 75014 Paris, France \\ ${ }^{\mathrm{f}}$ Université de Paris, Faculté de Médecine, 75006 Paris, France \\ g UMR1058 Inserm, 34000 Montpellier, France \\ h Department of Bacteriology-Virology, CHU Montpellier, 34000 Montpellier, France
}

\section{A R T I C L E I N F O}

\section{Keywords:}

Tuberculosis

Latent tuberculosis

Multidetector computed tomography

Interferon-gamma release tests

\begin{abstract}
A B S T R A C T
Purpose: The purpose of this study was to evaluate the capabilities of chest computed tomography (CT) in distinguishing between active and latent tuberculosis in patients positive for interferon-gamma release assay (IGRA) testing, and to compare the performance of CT with that of quantitative IGRA testing in a low incidence setting.

Materials and methods: Patients with latent or active tuberculosis define by an IGRA positive test were retrospectively recruited. Sensitivity, specificity and accuracy were determined for CT variables and quantitative IGRA results. Final diagnosis of active tuberculosis was based on clinical data and microbiological culture. Univariable and multivariable analyses were performed using logistic regression model to identify CT variables associated with the diagnosis of active tuberculosis.

Results: A total of 92 patients with positive IGRA results who underwent CT examination were included. There were 54 men and 38 women with a mean age of $53.5 \pm 18$ (SD) years (range: 40-68 years). Of them, 22 patients (24\%) had positive Mycobacterium tuberculosis culture and 70 (76\%) had latent tuberculosis. Among CT variables, consolidation had the greatest sensitivity (77\%; 95\%CI: 60-95\%) and "tree-in-bud" the greatest specificity (97\%; 95\% CI: 93-100\%) for the diagnosis of active tuberculosis. At univariable analysis "tree-in-bud", splenic calcification and non-calcified lung nodules were the significant variables independently associated with active tuberculosis. At multivariable analysis, the adjusted odds ratio of “tree-in-bud" was 42.91 (95\% CI: 5.62-327.42). Using an optimal threshold of 51 spots, quantitative IGRA yielded 64\% sensitivity (95\% CI: $44-84 \%$ ) and $61 \%$ specificity (95\% CI: $50-73 \%$ ) for the diagnosis of active tuberculosis.

Conclusions: In a low incidence setting, chest CT, especially when "tree-in-bud" pattern is present, is superior to quantitative IGRA testing to identify patients with active tuberculosis among those with positive IGRA testing.
\end{abstract}

Abbreviations: CI, Confidence interval; CT, Computed tomography; HR, Hazard ratio; IGRA, Interferon-gamma release assay; SD, Standard deviation; WHO, World health organization.

* Corresponding author at: Department of Radiology, CHU Montpellier, avenue Doyen Gaston Giraud, 34000 Montpellier, France.

E-mail address: s-bommart@chu-montpellier.fr (S. Bommart).

\section{Introduction}

Although chest X-ray examination remains the only recommended imaging modality in the management of tuberculosis according to current guidelines [1], chest computed tomography (CT) can be useful for assessing differential diagnosis in symptomatic patients. Chest CT is also useful in the investigation of contact subjects of known patients with tuberculosis and in 
immunocompromised patients because it is more sensitive than chest X-ray [2,3].

However, the interpretation of chest CT abnormalities in patients with a history of tuberculosis is challenging, especially when no prior CT examination is available, and accurate criteria are needed to distinguish active tuberculosis from sequelae. The question is even more complex when considering the concept of latent tuberculosis. The WHO-definition of latent tuberculosis is based on the existence of a specific immune response with an absence of clinical signs. Chest X ray is included within the "clinical signs" cited by the $\mathrm{WHO}$, contrary to $\mathrm{CT}$ whose role remains controversial as a diagnostic tool for tuberculosis diagnosis [4].

As sputum smear microscopy is not sensitive enough, bacterial culture remains the gold standard for confirmation of Mycobacterium tuberculosis infection, although final results may take up to 12 weeks. During this phase, differentiating between latent and active tuberculosis infection is challenging and is likely to delay the start of specific care or lead to prolonged unnecessary airborne precautions. Although other biomarkers, such as interferon-gamma release assay (IGRA) add important information in well-identified situations such as contact with an active tuberculosis patient or before immunosuppression, positivity of IGRA testing alone is insufficient to justify specific treatment, especially in low incidence countries [5,6]. Sputum nucleic acid amplification tests are used as additional and/or alternative test when culture is not available $[7,8]$. Neither test provides a definitive diagnosis, but they could replace the microscopic smear as the first-line diagnostic test in areas with an intermediate tuberculosis burden. [9].

Until now, chest CT criteria is only used to describe latent and active tuberculosis [10-14]. Patterns of disease activity in tuberculosis have been highlighted and compared to tuberculosis culture but no study evaluated the diagnostic performance of chest CT to distinguish between active and latent tuberculosis in patients with a positive IGRA testing.

The purpose of this study was to evaluate the capabilities of chest $\mathrm{CT}$ in distinguishing between active and latent tuberculosis in patients with IGRA positive tests, and to compare the performance of CT with that of quantitative IGRA.

\section{Materials and methods}

\subsection{Study design and population}

Our institutional review board (AFSSAPS 2010-A00422-37, SudMéditerranée) approved this study and written informed consent was obtained from all participants. CT examinations of patients with clinically suspected chest tuberculosis and positive IGRA results who were recruited between December 2008 and October 2012 were retrospectively analyzed. Inclusion and exclusion criteria are given in Table 1. Chest CT was performed either to help chest $\mathrm{X}$-ray interpretation or performed because of unspecific symptoms such as cough, fever or expectoration. Only patients with positive IGRA testing (i.e., > 7 spots/100000) were included. Fig. 1 describes the study flow chart of patients. Smear cultures were concomitantly initiated on three consecutive days and processed according to best standards. In some patients broncho-alveolar lavage and bronchial aspirates were also sampled and processed for tuberculosis-culture.

\subsection{CT protocol}

Chest CT examinations were performed with or without contrast media injection in the supine position using various multidetector CT scanners. Images were acquired in our department using a lightspeed ${ }^{\circledR}$ VCT unit (General Electric Healthcare) at $120 \mathrm{kV}$
Table 1

Inclusion and exclusion criteria.

\begin{tabular}{|c|c|}
\hline Inclusion criteria & Exclusion criteria \\
\hline Male or female & Age $<18$ years \\
\hline \multicolumn{2}{|l|}{ Age $>18$ years } \\
\hline $\begin{array}{l}\text { Clinically suspected tuberculosis ('or' } \\
\text { should be read between each item) }\end{array}$ & $\begin{array}{l}\text { Asymptomatic patient or no } \\
\text { clinical information available }\end{array}$ \\
\hline \multicolumn{2}{|l|}{ Persistent cough $>2$ weeks } \\
\hline \multicolumn{2}{|l|}{ Fever } \\
\hline \multicolumn{2}{|l|}{ Hemoptysis } \\
\hline \multicolumn{2}{|l|}{ Nocturnal sweats } \\
\hline \multicolumn{2}{|l|}{ Loss of weight, anorexia } \\
\hline \multicolumn{2}{|l|}{ Asthenia } \\
\hline \multicolumn{2}{|l|}{$\begin{array}{l}\text { Contact with a subject definitively } \\
\text { diagnosed with active tuberculosis }\end{array}$} \\
\hline $\begin{array}{l}\text { All consecutive patients who } \\
\text { underwent IGRA in our institution } \\
\text { between Dec } 2008 \text { and oct } 2012\end{array}$ & $\begin{array}{l}\text { IGRA test failed or below the } \\
\text { quality control standards }\end{array}$ \\
\hline \multirow[t]{2}{*}{$\begin{array}{l}\text { Chest CT examination of acceptable } \\
\text { quality }\end{array}$} & $\begin{array}{l}\text { Incomplete chest } \mathrm{CT} \\
\text { examination }\end{array}$ \\
\hline & $\begin{array}{l}\mathrm{CT} \text { image thickness }>1.25 \mathrm{~mm} \\
\text { or non-contiguous slices }\end{array}$ \\
\hline \multirow[t]{2}{*}{ Affiliated to the French health coverage } & Not protected by law \\
\hline & Deprived of liberty \\
\hline
\end{tabular}

CT indicates computed tomography. IGRA indicates Interferon-gamma release assay.

with automated tube current modulation and a maximal tube current set at $300 \mathrm{~mA}, 0.5 \mathrm{~s}$ tube rotation; $64 \times 0.6 \mathrm{~mm}$ collimation and 0.9 increment. Images were reconstructed with a 512 matrix, $0.625 \mathrm{~mm}$ and $1.25 \mathrm{~mm}$ reconstruction slice thicknesses using a high and standard frequency kernel. Acquisition field-ofview ranged from 320 - to $380 \mathrm{~mm}$ depending on patient habitus.

\subsection{Quantitative IGRA test}

Immune reactivity to Mycobacterium tuberculosis was tested using the ELISA assay, T-spot tuberculosis (Oxford Immunotec Ld.) which reveals the specific interferon-gamma production in response to stimulation by specific mycobacterial proteins.

\subsection{Image analysis}

CT examinations were randomly assigned and read on a workstation (ADW 4.6, GE healthcare) by two readers (S.B., A.B.) with 8 - and 10-year experience in chest imaging respectively, blinded to the results of IGRA and clinical data. Images were reviewed using a lung (width 1600 Hounsfield unit [HU]; center -600 HU) and a mediastinal window setting (width $440 \mathrm{HU}$; center $40 \mathrm{HU}$ ). The readers had to report the presence of one or more abnormalities, among the following items defined according to the Fleischner society glossary terms [15]. These included: (i), randomly distributed calcified and non-calcified nodules (nodules fulfilling intra pulmonary lymph node criteria (i.e., sub pleural or perifissural location, triangular shape [ $<1 \mathrm{~cm}$ in size were not considered here]) (Fig. 2) [10]; (ii), centrilobular nodules [10,14]; (iii), branching linear opacities ("tree-in-bud" pattern) $[10,14]$; (iv), cavities; ( $v$ ), consolidations [11,12]; (vi), localized fibrosis [10]; (vii), pleural [11] or (viii), pericardial effusions [11]; (ix), enlarged mediastinal lymph nodes (i.e., > $10 \mathrm{~mm}$ short axis) [10]; $(x)$, splenic [16] or ( $x i)$, hepatic calcified nodules [16]. Spatial distribution in the ten right and left segments of the Boyden classification was also determined for each sign. The two readers used a structured report including a description for each abnormality of the pre-defined list. When discordant reading was obtained, a consensus opinion was reached by the two readers during a final reading session. The results of the consensus reading were used for further analysis. 


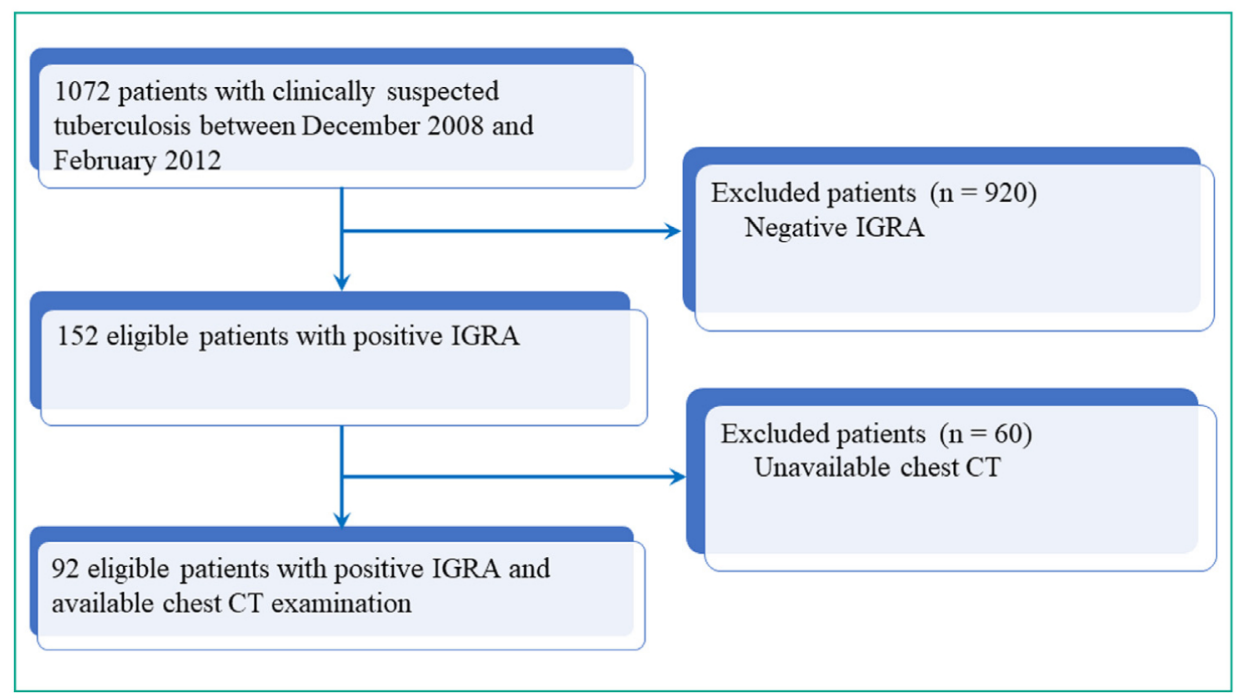

Fig. 1. Flowchart shows patient inclusion in the study. CT indicates computed tomography; IGRA indicates interferon-gamma release assay.

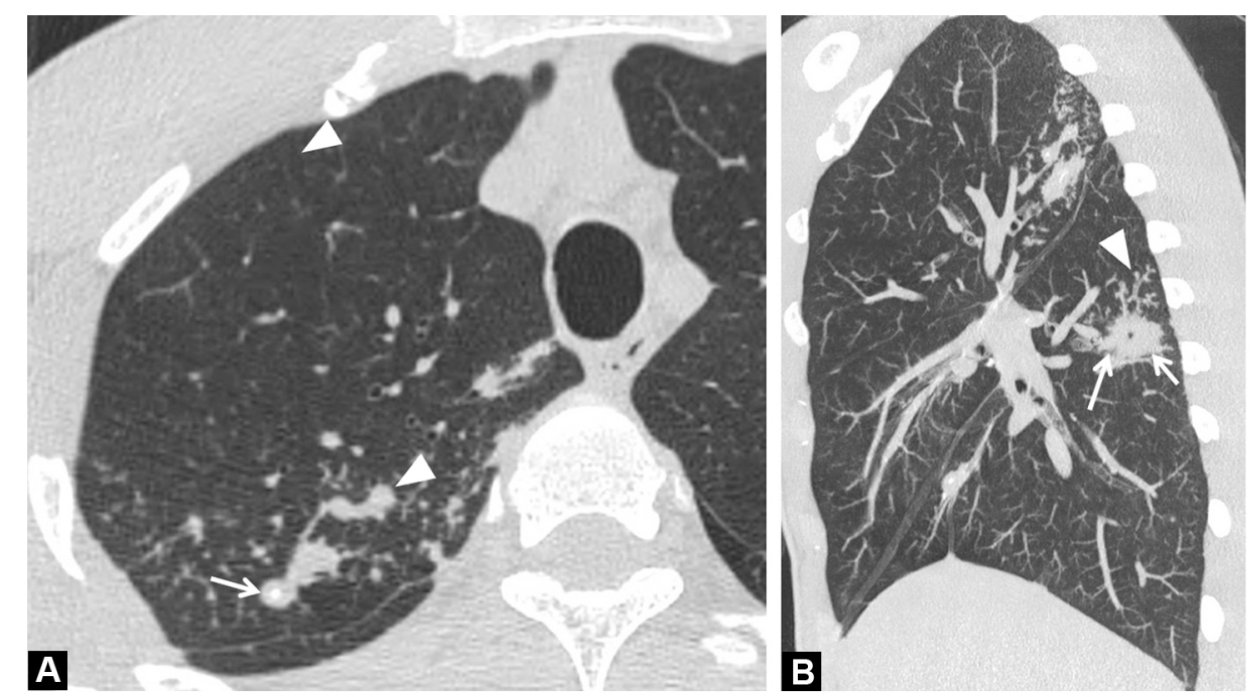

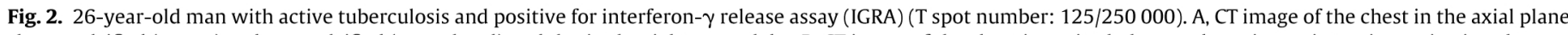

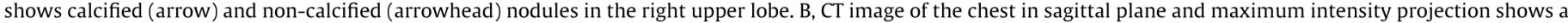
mass (arrow) with a small cavity and micronodules (arrowheads) displaying the "tree in bud" pattern.

\subsection{Standard of reference}

CT findings were compared to quantitative IGRA results (number of interferon- $\gamma$ producing cells also called T-spot) and final diagnoses for active tuberculosis were established using clinical data and microbiological culture.

Immune reactivity to Mycobacterium tuberculosis (IGRA testing) was tested using the ELISA assay, T-spot tuberculosis (Oxford Immunotec $\mathrm{Ld}$.) which reveals the specific interferon- $\gamma$ production in response to stimulation by specific mycobacterial proteins.

Latent tuberculosis infection was defined by an IGRA positive test and an absence of tuberculosis-culture positivity at 12 weeks after sample gathering, or absence of clinical symptoms at twelve months follow-up. Active tuberculosis infection was defined by a positive IGRA test and a positive tuberculosis culture.

\subsection{Statistical analysis}

All analyses were performed using SAS version 6.1 (SAS Institute). Qualitative data were reported as raw numbers, proportions and percentages. Quadratic kappa $(\kappa)$ coefficients were calculated to assess interobserver agreement between the two readers before the final consensus reading and reported with their 95\% confidence intervals (CIs) [17]. The threshold of the number of T-spots to differentiate between patients with active tuberculosis and those with latent tuberculosis was determined using the Youden index. Quantitative variables in patients with active tuberculosis were compared to those in patients with latent tuberculosis using the Mann-Whitney test. The frequency and location of each CT sign in active and latent tuberculosis were compared using the $\mathrm{Chi}^{2}$ test. The $P$-values were adjusted using the Holm-Bonferroni method. The corresponding sensitivities, specificities and accuracies were calculated along with their 95\% CIs.

To identify CT variables associated with the diagnosis of active tuberculosis, categorical variables were entered into univariable analysis using a conditional logistic regression model. To take into account confounders, multivariable analysis was performed using a logistic regression model with forward stepwise selection of covariables and adjusted for age. Correlations between all variables were searched for. In case of a strong correlation between two variables, 
Table 2

Inter-observer agreement for chest CT variables.

\begin{tabular}{|c|c|c|c|c|}
\hline Variable & Observer 1 & Observer 2 & Kappa & $95 \% \mathrm{CI}$ \\
\hline Centrilobular nodules & $16(16 / 92 ; 17.4 \%)$ & $5(5 / 92 ; 5.4 \%)$ & 0.32 & $0.06-0.58$ \\
\hline Tree-in-bud & $11(11 / 92 ; 11.9 \%)$ & $26(26 / 92 ; 40.2 \%)$ & 0.51 & $0.31-0.71$ \\
\hline Cavity & $8(8 / 92 ; 8.7 \%)$ & $12(12 / 92 ; 13 \%)$ & 0.66 & $0.41-0.91$ \\
\hline Consolidation & $37(37 / 92 ; 40.2 \%)$ & $37(37 / 92 ; 40.2 \%)$ & 0.68 & $0.53-0.83$ \\
\hline Non-calcified nodule & $36(36 / 92 ; 39.1 \%)$ & $35(35 / 92 ; 38 \%)$ & 0.51 & $0.33-0.69$ \\
\hline Calcified nodule & $40(40 / 92 ; 43.5 \%)$ & $26(26 / 92 ; 28.3 \%)$ & 0.53 & $0.36-0.70$ \\
\hline Pleural effusion & $14(14 / 92 ; 15.2 \%)$ & $17(17 / 92 ; 18.5 \%)$ & 0.88 & $0.75-1$ \\
\hline Pericardial effusion & $5(5 / 92 ; 5.4 \%)$ & $8(8 / 92 ; 8.7 \%)$ & 0.75 & $0.48-1$ \\
\hline Hepatic calcified nodules & $10(10 / 92 ; 10.9 \%)$ & $13(13 / 92 ; 14.1 \%)$ & 0.65 & $0.42-0.89$ \\
\hline Splenic calcified nodules & $5(5 / 92 ; 5.4 \%)$ & $7(7 / 92 ; 7.6 \%)$ & 0.64 & $0.32-0.97$ \\
\hline Enlarged mediastinal lymph node & $21(21 / 92 ; 22.8 \%)$ & $38(38 / 92 ; 41.3 \%)$ & 0.43 & $0.25-0.61$ \\
\hline
\end{tabular}

Data are expressed as raw numbers; numbers in parentheses are proportions followed by percentages.

Table 3

Comparison of CT findings for categorical criteria between 22 patients with active tuberculosis and 70 patients with latent tuberculosis.

\begin{tabular}{|c|c|c|c|}
\hline Variable & Group 1Active TB $(n=22)$ & Group 2Latent TB $(n=70)$ & $P$ value \\
\hline Centrilobular nodules & $10(10 / 22 ; 45 \%)$ & $6(6 / 70 ; 8.6 \%)$ & 0.002 \\
\hline "Tree-in-bud" & $9(9 / 22 ; 40.9 \%)$ & $2(2 / 70 ; 2.9 \%)$ & 0.002 \\
\hline Cavity & $5(5 / 22 ; 22.7 \%$ & $3(3 / 70 ; 4.3 \%)$ & 0.234 \\
\hline Consolidation & $17(17 / 22 ; 77.3 \%)$ & $20(20 / 70 ; 28.6 \%)$ & 0.002 \\
\hline Non-calcified nodule & $14(14 / 22 ; 64 \%)$ & $22(22 / 70 ; 32 \%)$ & 0.126 \\
\hline Calcified nodule & $11(11 / 22 ; 52 \%)$ & $29(29 / 70 ; 42 \%)$ & $>0.999$ \\
\hline Pleural effusion & $4(4 / 22 ; 19 \%)$ & $10(10 / 70 ; 14 \%)$ & $>0.999$ \\
\hline Pericardial effusion & $2(2 / 22 ; 9.5 \%)$ & $3(3 / 70 ; 4.3 \%)$ & $>0.999$ \\
\hline Hepatic calcified nodules & $1(1 / 22 ; 4.7 \%)$ & $9(9 / 70 ; 12.8 \%)$ & $>0.999$ \\
\hline Splenic calcified nodules & $3(3 / 22 ; 15 \%)$ & $2(2 / 70 ; 2.8 \%)$ & 0.876 \\
\hline Enlarged mediastinal lymph node & $7(7 / 22 ; 33 \%)$ & $14(14 / 70 ; 20 \%)$ & $>0.999$ \\
\hline
\end{tabular}

TB indicates tuberculosis. Bold indicates significant $P$ value. Numbers are raw numbers; numbers in parentheses are proportions followed by percentages.

a Corresponding to the Holm adjusted $P$ value.

one or other variables was included in the multivariable model. All tests were two-tailed and statistical significance was set at a $P$-value $<0.05$.

Significant chest $\mathrm{CT}$ variables in the multivariable model were further compared between upper and lower lung segments (numbers 1, 2, 3 and 6 versus others) using the Fisher exact test.

\section{Results}

\subsection{Patient characteristics}

The final cohort study consisted of 92 patients with positive IGRA who underwent chest CT. There were 54 men and 38 women with a mean age of $53.5 \pm 18$ (SD) years (range: 40-68 years). Among them, 22 patients (13 men, 9 women; mean age, $40.5 \pm 19$ [SD] years; age range: $29-53$ years) had active tuberculosis. Seventy patients (41 men, 29 women; mean age, $57.0 \pm 24$ [SD] years; age range: $42-70$ years) were considered having latent tuberculosis infection. The diagnosis of latent tuberculosis was based on negative 12 -week culture results and a 12 -month negative clinical follow-up.

\subsection{Description of CT patterns and quantitative IGRA in latent and active tuberculosis}

Inter-observer agreement ranged from fair to almost perfect (Table 2). Kappa values obtained for centrilobular nodules was 0.32 (95\% CI: 0.06-0.58) and 0.88 (95\% CI: 0.75-1) for pleural effusion.

On CT, centrilobular nodules $(P=0.002)$, "tree-in-bud" pattern $(P=0.002)$ as well as consolidation $(P=0.002)$ were more frequently observed in patients with active tuberculosis than in those with latent tuberculosis. Table 3 shows the distribution of CT findings for the two groups of patients.

The sensitivity, specificity and accuracy of each CT variable for the diagnosis of active tuberculosis are reported in Table 4.
Alveolar consolidation showed greatest sensitivity for the diagnosis of active tuberculosis (77\%; 17/22; 95\% CI: 60--95\%) followed by non-calcified lung nodules (64\%; $14 / 22 ; 95 \% \mathrm{CI}$ : 43-84\%). Specificity of "tree-in-bud" for the diagnosis of active tuberculosis ranked first as well as splenic calcified nodules (97\%; 68/70; 95\% CI: 93-100\% for both), followed by cavities (96\%; 67/70; 95\% CI: 0.91-1.00).

IGRA sensitivity for active tuberculosis was $64 \%$ (14/22; $95 \%$ CI: $44-84 \%$ ) and specificity $61 \%$ (43/70; 95\% CI: 50-73\%) using an optimal threshold of 51 spots.

At multivariable logistic regression analysis (Table 5 ), the presence of tree-in-bud (OR, 25.5; 95\% CI: 4.9-132.8) $(P<0.001)$, non-calcified nodules (OR, 3.7; 95\% CI: 1.3-10) $(P=0.012)$, lymph nodes enlargement (OR, 3.66; 95\% CI: 1.34-10.01) $(P=0.17)$ and splenic calcified nodules (OR, 5.9; 95\% CI: 0.9-38.2) $(P=0.062)$ were independently associated with active tuberculosis. Adjusted for age, OR of tree-in-bud in the multivariate analysis was 42.91 (95\% CI: 5.62-327.42).

The location of non-calcified nodules and tree-in-bud in the upper lung (segments 1, 2, 3 and 6 vs. others) did not improve the diagnosis of disease activity ( $P=0.19$ and $P=0.189$, respectively).

\section{Discussion}

In this study, the performance of chest CT in discriminating between latent and active tuberculosis in a population positively screened by IGRA was investigated and then compared to the discriminative value of the quantitative IGRA. Our results show that "tree-in-bud" and non-calcified nodules on CT significantly and better diagnose active tuberculosis than quantitative IGRA.

Despite advances in health care, Mycobacterium tuberculosis infection remains a major public health burden, worsened in the past decade by increased levels of immunosuppression in the population and antibiotic resistance [18]. The WHO does not recommend chest CT or IGRA test to assess tuberculosis in endemic countries. 
Table 4

Sensitivity, specificity and accuracy of each CT variables for the diagnosis of active tuberculosis vs. latent tuberculosis.

\begin{tabular}{|c|c|c|c|c|c|c|c|}
\hline Variable & $\mathrm{TN}$ & $\mathrm{TP}$ & FN & FP & Sensitivity & Specificity & Accuracy \\
\hline Centrilobular nodules & 64 & 10 & 12 & 6 & $\begin{array}{l}45(10 / 22) \\
{[25-66]}\end{array}$ & $\begin{array}{l}91(64 / 70) \\
{[85-98]}\end{array}$ & $\begin{array}{l}80(74 / 92) \\
{[72-88]}\end{array}$ \\
\hline "Tree in bud" & 68 & 9 & 13 & 2 & $\begin{array}{l}41(9 / 22) \\
{[20-61]}\end{array}$ & $\begin{array}{l}97(68 / 70) \\
{[93-100]}\end{array}$ & $\begin{array}{l}84(77 / 92) \\
{[76-91]}\end{array}$ \\
\hline Cavity & 67 & 5 & 19 & 3 & $\begin{array}{l}23(5 / 22) \\
{[5-40]}\end{array}$ & $\begin{array}{l}96(67 / 70) \\
{[91-100]}\end{array}$ & $\begin{array}{l}78(72 / 92) \\
{[70-87]}\end{array}$ \\
\hline Consolidation & 50 & 17 & 5 & 20 & $\begin{array}{l}77(17 / 22) \\
{[60-95]}\end{array}$ & $\begin{array}{l}71(50 / 70) \\
{[61-82]}\end{array}$ & $\begin{array}{l}73(67 / 92) \\
{[64-82]}\end{array}$ \\
\hline Non-calcified nodules & 48 & 14 & 8 & 22 & $\begin{array}{l}64(14 / 22) \\
{[43-84]}\end{array}$ & $\begin{array}{l}69(48 / 70) \\
{[58-79]}\end{array}$ & $\begin{array}{l}67(62 / 92) \\
{[58-77]}\end{array}$ \\
\hline Calcified nodules & 41 & 11 & 11 & 29 & $\begin{array}{l}50(11 / 22) \\
{[29-71]}\end{array}$ & $\begin{array}{l}59(41 / 70) \\
{[47-70]}\end{array}$ & $\begin{array}{l}56(52 / 92) \\
{[46-66]}\end{array}$ \\
\hline Pleural effusion & 60 & 4 & 18 & 10 & $\begin{array}{l}18(4 / 22) \\
{[2-34]}\end{array}$ & $\begin{array}{l}0.86(60 / 70) \\
{[77-94]}\end{array}$ & $\begin{array}{l}70(64 / 92) \\
{[60-79]}\end{array}$ \\
\hline Pericardial effusion & 67 & 2 & 20 & 3 & $\begin{array}{l}9(2 / 22) \\
{[3-21]}\end{array}$ & $\begin{array}{l}96(67 / 70) \\
{[91-100]}\end{array}$ & $\begin{array}{l}75(69 / 92) \\
{[66-84]}\end{array}$ \\
\hline Hepatic calcified nodules & 61 & 1 & 21 & 9 & $\begin{array}{l}4(1 / 22) \\
{[4-13]}\end{array}$ & $\begin{array}{l}87(61 / 70) \\
{[79-95]}\end{array}$ & $\begin{array}{l}67(62 / 92) \\
{[58-77]}\end{array}$ \\
\hline Splenic calcified nodules & 68 & 3 & 19 & 2 & $\begin{array}{l}14(3 / 22) \\
{[1-28]}\end{array}$ & $\begin{array}{l}97(68 / 70) \\
{[93-100]}\end{array}$ & $\begin{array}{l}77(71 / 92) \\
{[69-86]}\end{array}$ \\
\hline Enlarged mediastinal lymph nodes & 56 & 7 & 15 & 14 & $\begin{array}{l}32(7 / 22) \\
{[12-51]}\end{array}$ & $\begin{array}{l}80(56 / 70) \\
{[71-89]}\end{array}$ & $\begin{array}{l}68(63 / 92) \\
{[59-78]}\end{array}$ \\
\hline
\end{tabular}

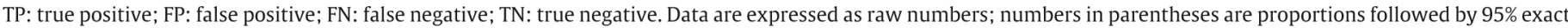
confidence intervals in brackets.

Table 5

Multivariable analysis for chest CT signs associated with active tuberculosis.

\begin{tabular}{|c|c|c|c|c|}
\hline Variable & OR $[95 \% \mathrm{Cl}]$ & $P$ value & Adjusted OR [95\% CI] & $P$ value \\
\hline Centrilobular nodules & $9.70[2.93-32.11]$ & $<0.001$ & & \\
\hline Tree-in-bud & $25.50[4.90-132.84]$ & $<0.001$ & $42.91[5.62-327.42]$ & $<0.001$ \\
\hline Cavity & $6.57[1.43-30.24]$ & 0.016 & & \\
\hline Consolidation & $8.50[2.76-26.15]$ & $<0.001$ & & \\
\hline Non-calcified nodule & $3.66[1.34-10.01]$ & 0.012 & $5.16[1.09-24.33]$ & 0.034 \\
\hline Calcified nodules & $1.52[0.57-4.05]$ & 0.405 & & \\
\hline Pleural effusion & $1.41[0.39-5.07]$ & 0.597 & & \\
\hline Pericardial effusion & $2.32[0.36-14.88]$ & 0.376 & & \\
\hline Hepatic calcified nodules & $0.3[0.04-2.95]$ & 0.335 & & \\
\hline Splenic calcified nodules & $5.91[0.91-38.22]$ & 0.062 & 14.49 [1.30-162.09] & 0.044 \\
\hline Enlarged mediastinal lymph node & $2.17[0.72-6.53]$ & 0.170 & $2.05[0.44-9.49]$ & 0.361 \\
\hline
\end{tabular}

OR: odds ratio. $95 \% \mathrm{CI}=95 \%$ exact confidence interval. Bold indicates significant $P$ value.

Nonetheless, the diagnosis of tuberculosis can be challenging in low prevalence areas such as in Europe or in the USA and induces important consequences on clinical outcomes. Chest X-ray is advocated by the WHO as a diagnostic tool but some abnormalities such as ẗree-in-budp̈attern may be missed [19]. Although the use of chest CT for the diagnosis of tuberculosis is still controversial, it is clearly indicated in most clinical situations where tuberculosis is uncertain. Moreover, CT can also help for lung lesions sampling for tuberculosis culture using percutaneous biopsy in doubtful situations [20].

Tree-in-bud is a frequent lung lesion pattern. Bronchiolar obstruction and alveolar filling are thought to explain this phenomenon. Hatipoğlu et al. also reported a relationship between "tree-in-bud" on CT and active tuberculosis [10]. "Tree-in-bud" has also been described as a frequent manifestation of lung infection and small airway diseases $[21,22]$. This sign had a greater specificity in our study for the diagnosis of active tuberculosis than quantitative IGRA test.

Unlike Yeh et al. who developed a CT scoring system to discriminate between tuberculosis infection and other pulmonary lesions, upper zone involvement was not identified as a finding associated with active tuberculosis in this study [12]. This can be explained by the fact that tuberculosis infection was not compared to other causes of parenchymal diseases. The comparison in this study was only made between active and latent tuberculosis, which can both occur simultaneously in the same lung segments.
Our study has limitations. First, this retrospective analysis from a single cohort included only 92 patients with latent or active tuberculosis. The sample size of some CT patterns such as lymph node enlargement or extra thoracic calcification suggestive of granuloma could limit the conclusions regarding these signs. Additionally, CT protocol, and especially low dose protocol may also influence imaging presentation, so that our findings should be confirmed from a validation cohort using standardized CT parameters $[23,24]$.

In conclusion, the results of this study highlight the added value of chest CT to the diagnosis of active tuberculosis in patients with positive IGRA showing that a "tree-in-bud" pattern has greater specificity for disease activity than quantitative IGRA testing. These findings should however be confirmed in a prospective validation cohort to improve the early detection of active tuberculosis in IGRA positive populations.

\section{Human rights}

The authors declare that the work described has been carried out in accordance with the Declaration of Helsinki of the World Medical Association revised in 2013 for experiments involving humans.

\section{Informed consent and patient details}

Institutional ethic committee approval was obtained. Written informed consent was obtained from the patients. The authors 
declare that this report does not contain any personal information that could lead to the identification of the patients. The authors also confirm that the personal details of the patients have been removed.

\section{Funding}

This work did not receive any grant from funding agencies in the public, commercial, or not-for-profit sectors.

\section{Authors' contributions}

All authors attest that they meet the current International Committee of Medical Journal Editors (ICMJE) criteria for Authorship.

\section{CRediT authorship contribution statement}

Sébastien Bommart: investigation, writing, writing - review \& editing original draft preparation, vizualization. Jéremy Charriot: writing - review \& editing original draft preparation, visualization. Nicolas Nagot: methodology, formal analysis, data curation. Hélène Vernhet-Kovacsik: writing - review \& editing. Marie Pierre Revel: writing - review \& editing original draft preparation. Clement Boissin: investigation. Arnaud Bourdin: conceptualization, writing - review \& editing original draft preparation, visualization. Edouard Tuaillon: investigation, supervision, validation.

\section{Disclosure of interest}

The authors declare that they have no competing interest.

\section{References}

[1] WHO | Global tuberculosis report 2018 [Internet]. WHO. http://www.who.int/tb/publications/global_report/en/.

[2] Lee SW, Jang YS, Park CM, Kang HY, Koh WJ, Yim JJ, et al. The role of chest CT scanning in tuberculosis outbreak investigation. Chest 2010;137:1057-64.

[3] Schluger NW. CT scanning for evaluating contacts of tuberculosis patients: ready for prime time? Chest 2010:137:1011-3.

[4] Wallis RS, Kim P, Cole S, Hanna D, Andrade BB, Maeurer M, et al. Tuberculosis biomarkers discovery: developments, needs, and challenges. Lancet Infect Dis 2013:13:362-72.

[5] Hermansen TS, Lillebaek T, Langholz Kristensen K, Andersen PH, Ravn P. Prognostic value of interferon- $\gamma$ release assays, a population-based study from a tuberculosis low-incidence country. Thorax 2016;71:652-8.
[6] Jung JY, Lim JE, Lee H-J, Kim YM, Cho S-N, Kim SK, et al. Questionable role of interferon- $\gamma$ assays for smear-negative pulmonary tuberculosis in immunocompromised patients. J Infect 2012;64:188-96.

[7] Blakemore R, Nabeta P, Davidow AL, Vadwai V, Tahirli R, Munsamy V, et al. A multisite assessment of the quantitative capabilities of the Xpert Mtuberculosis/RIF assay. Am J Respir Crit Care Med 2011;184:1076-84.

[8] Ngabonziza JCS, Ssengooba W, Mutua F, Torrea G, Dushime A, Gasana M, et al. Diagnostic performance of smear microscopy and incremental yield of Xpert in detection of pulmonary tuberculosis in Rwanda. BMC Infect Dis 2016;16:660.

[9] Lee H-S, Kee S-J, Shin J-H, Kwon Y-S, Chun S, Lee JH, et al. Xpert Mtuberculosis/RIF assay as a substitute for smear microscopy in an intermediate-burden setting. Am J Respir Crit Care Med 2018;199:784-94.

[10] Hatipoğlu ON, Osma E, Manisali M, Uçan ES, Balci P, Akkoçlu A, et al. High resolution computed tomographic findings in pulmonary tuberculosis. Thorax 1996;51:397-402.

[11] Jeong YJ, Lee KS. Pulmonary tuberculosis: up-to-date imaging and management. AJR Am J Roentgenol 2008;191:834-44.

[12] Yeh JJ, Chen SC-C, Teng W-B, Chou C-H, Hsieh S-P, Lee T-L, et al. Identifying the most infectious lesions in pulmonary tuberculosis by high-resolution multidetector computed tomography. Eur Radiol 2010;20:2135-45.

[13] Nguyen M-VH, Jenny-Avital ER, Burger S, Leibert EM, Achkar JM. Clinical and radiographic manifestations of sputum culture-negative pulmonary tuberculosis. PLoS One 2015;10 [e0140003].

[14] Ko JM, Park HJ, Kim CH, Song SW. The relation between CT findings and sputum microbiology studies in active pulmonary tuberculosis. Eur J Radiol 2015;84:2339-44.

[15] Hansell DM, Bankier AA, MacMahon H, McLoud TC, Müller NL, Remy J. Fleischner Society: glossary of terms for thoracic imaging. Radiology 2008;246:697-722.

[16] Schininà V, Albarello F, Cristofaro M, Di Stefano F, Fusco N, Cuzzi G, et al Diagnostic imaging of hepatic tuberculosis: case series. Int J Tuberc Lung Dis 2018;22:779-87.

[17] Benchoufi M, Matzner-Lober E, Molinari N, Jannot AS, Soyer P. Interobserver agreement issues in radiology. Diagn Interv Imaging 2020;101:639-41.

[18] Murray CJL, Ortblad KF, Guinovart C, Lim SS, Wolock TM, Roberts DA, et al. Global, regional, and national incidence and mortality for HIV, tuberculosis, and malaria during 1990-2013: a systematic analysis for the Global Burden of Disease Study 2013. Lancet 2014;384:1005-70.

[19] Eisenberg RL, Pollock NR. Low yield of chest radiography in a large tuberculosis screening program. Radiology 2010;256:998-1004.

[20] Brioulet J, David A, Sagan C, Cellerin L, Frampas E, Morla O. Percutaneous CTguided lung biopsy for the diagnosis of persistent pulmonary consolidation. Diagn Interv Imaging 2020;101:727-32.

[21] Shimon G, Yonit W-W, Gabriel I, Naama BR, Nissim A. The "tree-in-bud" pattern on chest CT: radiologic and microbiologic correlation. Lung 2015;193:823-9.

[22] Okada F, Ando Y, Yoshitake S, Ono A, Tanoue S, Matsumoto S, et al. Clini$\mathrm{cal} /$ pathologic correlations in 553 patients with primary centrilobular findings on high-resolution CT scan of the thorax. Chest 2007;132:1939-48.

[23] Beregi JP, Greffier J. Low and ultra-low dose radiation in CT: opportunities and limitations. Diagn Interv Imaging 2019;100:63-4.

[24] Greffier J, Boccalini S, Beregi JP, Vlassenbroek A, Vuillod A, Dupuis-Girod S, et al. CT dose optimization for the detection of pulmonary arteriovenous malformation (PAVM): a phantom study. Diagn Interv Imaging 2020;101:289-97. 